\title{
Enfrentamento religioso/espiritual em pessoas com câncer em quimioterapia: revisão integrativa da literatura
}

\author{
Fernanda Fernandes de Paiva Ribeiro Dantas Sousa ${ }^{1}$ \\ Susy Maria Feitosa de Melo Freitas ${ }^{2}$ \\ Ana Gesselena da Silva Farias ${ }^{3}$ \\ Maria da Conceição dos Santos Oliveira Cunha ${ }^{3}$ \\ Márcio Flávio Moura de Araújo ${ }^{4}$ \\ Vívian Saraiva Veras ${ }^{4}$
}

\begin{abstract}
Objetivou-se identificar as evidências científicas disponíveis acerca do enfrentamento religioso/espiritual utilizado por pessoas com câncer, em quimioterapia. Trata-se de revisão integrativa da literatura. Utilizou-se os descritores Quimioterapia e Espiritualidade. As bases de dados foram: LILACS, PUBMED, ScienceDirect, EMBASE, CINAHL e BDENF. Os estudos reconhecem a religião/espiritualidade como estratégia de enfrentamento positivo, ao contribuírem para a adesão ao tratamento e redução do estresse. O enfrentamento negativo foi associado à incidência de efeitos colaterais, sofrimento psicológico, ansiedade e depressão. Conclui-se que o reconhecimento da espiritualidade como estratégia de enfrentamento e a identificação das carências espirituais fornecem aos profissionais de enfermagem um melhor escopo de informações para o plano de cuidados e para uma assistência mais integral.
\end{abstract}

Descritores: Quimioterapia; Espiritualidade; Religião; Adaptação Psicológica; Enfermagem Oncológica.

\footnotetext{
${ }^{1}$ Enfermeira, Instituto do Câncer do Ceará, Fortaleza, CE, Brasil.

2 Mestranda, Universidade da Integração Internacional da Lusofonia Afro-Brasileira, Redenção,CE, Brasil. Enfermeira, Secretaria da Saúde de Caucaia, Caucaia, CE, Brasil.

${ }^{3}$ Mestranda, Universidade da Integração Internacional da Lusofonia Afro-Brasileira, Redenção,CE, Brasil.

${ }^{4} \mathrm{PhD}$, Professor Adjunto, Universidade da Integração Internacional da Lusofonia Afro-Brasileira, Redenção,CE, Brasil.
} 


\section{Religious/Spiritual Coping by People with Cancer Undergoing Chemotherapy: Integrative Literature Review}

The objective of this study was to identify the scientific evidence available regarding the religious/spiritual coping approach used by people with cancer undergoing chemotherapy. It is an integrative review of the literature. The descriptors "chemotherapy" and "spirituality" were used. The databases were: LILACS, PUBMED, ScienceDirect, EMBASE, CINAHL, and BDENF. The studies recognize religion/spirituality as a positive approach strategy, since it contributes to adherence to treatment and stress reduction. The negative effect was associated with the incidence of collateral effects, psychological suffering, anxiety, and depression. We concluded that the recognition of spirituality as a coping strategy and the identification of spiritual needs provide nursing professionals with a better scope of information for care planning and more comprehensive assistance.

Descriptors: Drug Therapy; Spirituality; Religion; Adaptation, Psychological; Oncology Nursing.

\section{Enfrentamiento religioso/espiritual en personas con cáncer en quimioterapia: revisión integrativa de la literatura}

Se objetivó identificar las evidencias científicas disponibles acerca del enfrentamiento religioso/espiritual utilizado por personas con cáncer, en quimioterapia. Se trata de revisión integrativa de la literatura. Se utilizó los descriptores Quimioterapia y Espiritualidad. Las bases de datos fueron: LILACS, PUBMED, ScienceDirect, EMBASE, CINAHL y BDENF. Los estudios reconocen la religión/espiritualidad como estrategia de enfrentamiento positivo, con el hecho de contribuir para la adhesión al tratamiento y reducción del estrés. El enfrentamiento negativo fue asociado a la incidencia de efectos colaterales, sufrimiento psicológico, ansiedad y depresión. Se concluye que el reconocimiento de la espiritualidad como estrategia de enfrentamiento y la identificación de las carencias espirituales suministran a los profesionales de enfermería un mejor objetivo de informaciones para el plan de atenciones y para una asistencia más integral.

Descriptores: Quimioterapia; Espiritualidad; Religión; Adaptación Psicológica; Enfermería Oncológica.

\section{Introdução}

O câncer é um grave problema de saúde pública, especialmente nos países em desenvolvimento. A Organização Mundial da Saúde (OMS), estima para o ano de 2025, uma incidência de 20 milhões de casos em todo o mundo. É a segunda causa de mortes por doenças crônicas no Brasil (16,3\%) onde, de acordo com a estimativa para o ano de 2016, serão registrados 596 mil novos casos da doença(1).

A quimioterapia é o tratamento mais comum na oncologia e durante esta modalidade terapêutica as pessoas podem apresentar alterações físicas e emocionais importantes, como dor, fadiga, apatia, 
perda de peso, alopecia, entre outros. Somados às internações prolongadas, todos estes fatores são causadores de desconforto, estresse e sofrimento, desencadeantes dos sentimentos de tristeza, indignação, angústia e medo da morte ${ }^{(2-3)}$.

O enfrentamento das alterações supracitadas demandam maior resiliência das pessoas com câncer e seus respectivos familiares. Neste contexto, é comum buscar na espiritualidade um sentido para a vivência dessa experiência, que pode ajudar pessoas com doenças crônicas através da ação de neurotransmissores que promovem sensação de bem estar, da atenuação da frequência cardíaca e respiratória, bem como dos níveis de ansiedade e cortisol, além de melhorar o sistema imunológico ${ }^{(2,4)}$.

O manejo de uma doença crônica como o câncer requer uma atenção individualizada, visto que esse momento pode ter significações diferenciadas para cada pessoa. Dessa maneira, apoiar o cuidado na espiritualidade pode ser imprescindível, uma vez que a mesma ocupa lugar de destaque na vida da maioria dos indivíduos. Fato é que a sua significância deve ser avaliada independente da sua adoção como ferramenta terapêutica, pois, o Coping Religioso/ Espiritual (CRE) pode desempenhar um papel tanto positivo como negativo, no manejo e enfrentamento da doença(5).

Pessoas religiosas apresentam maior capacidade para lidar com circunstâncias adversas da vida através da utilização do CRE. O Coping, ou enfrentamento, refere-se a um conjunto de estratégias comportamentais e cognitivas, utilizadas com o objetivo de enfrentar situações de estresse. Quando positivo, abrange estratégias que proporcionam efeito benéfico ao indivíduo, como procurar amor/ proteção de Deus ou maior conexão com forças transcendentais. O CRE negativo envolve estratégias que geram consequências prejudiciais, como por exemplo, redefinir o estressor como punição divina ${ }^{(5)}$.

O CRE é a resposta mais adaptativa ao estresse e inclui recursos como a resolução de problemas, planejamento e busca de apoio emocional. Enfrentamento de esquiva ou não adaptativo, por outro lado, refere-se principalmente aos métodos pelos quais os indivíduos tentam evitar enfrentar problemas mentalmente e/ou fisicamente, distanciando-se do estressor, incluindo estratégias tais como a negação e o uso de álcool/drogas ${ }^{(5-6)}$.

Existem estudos sobre a relação da religiosidade/espiritualidade na prevenção do uso de $\operatorname{drogas}^{(6)}$, qualidade de vida ${ }^{(7-8)}$, terapia hormonal(9) e radioterapia(10). De nosso conhecimento, não apurouse publicação que tenha analisado o papel do CRE em pessoas durante tratamento quimioterápico. Dessa forma, o objetivo desta revisão foi sintetizar o conhecimento gerado a partir dos resultados de outros estudos, e com base nesses, desenvolver generalizações sobre o impacto da religiosidade/ espiritualidade no enfrentamento de pessoas com câncer em quimioterapia.

\section{Método}

Trata-se de revisão integrativa da literatura, realizada durante os meses de maio e junho de 2016.

A pergunta norteadora do presente estudo foi: quais as evidências presentes na literatura acerca do enfrentamento religioso/espiritual utilizado por pessoas com câncer em quimioterapia?

Utilizou-se as bases de dados LILACS, EMBASE, PUBMED, CINAHL, Science Direct e BDENF. Utilizou-se os descritores controlados Quimioterapia (Chemotherapy/Quimioterapia) e Espiritualidade (Spirituality/Espiritualidad) e o operador booleano AND entre os termos.

Foram adotados os seguintes critérios para inclusão dos artigos: artigo original e artigos com resumos e textos completos disponíveis para análise, aqueles publicados nos idiomas inglês, português ou espanhol, entre os anos de 2005 e 2016. As buscas foram realizadas por três revisores, seguindo o mesmo procedimento, com o objetivo de validar os resultados encontrados.

Foram excluídos os estudos de revisão, teses e dissertações, relatos de casos informais, capítulos de livros, reportagens, editoriais e textos não científicos, além de artigos que não faziam referência ao objetivo do estudo.

A partir da leitura dos títulos e resumos de todas as publicações encontradas, e de acordo com os critérios de inclusão e exclusão definidos, obteve-se a seleção prévia dos artigos que integraram a amostra deste estudo. A amostra final foi de 11 artigos.

A Figura 1 exemplifica o número de estudos primários identificados em cada base de dados $(\mathrm{N})$. Os artigos que atenderam aos critérios de inclusão compuseram o número de artigos pré-selecionados (n). Assim, os artigos selecionados para o estudo, excluindo as repetições entre as bases de dados, compuseram o "N final". 


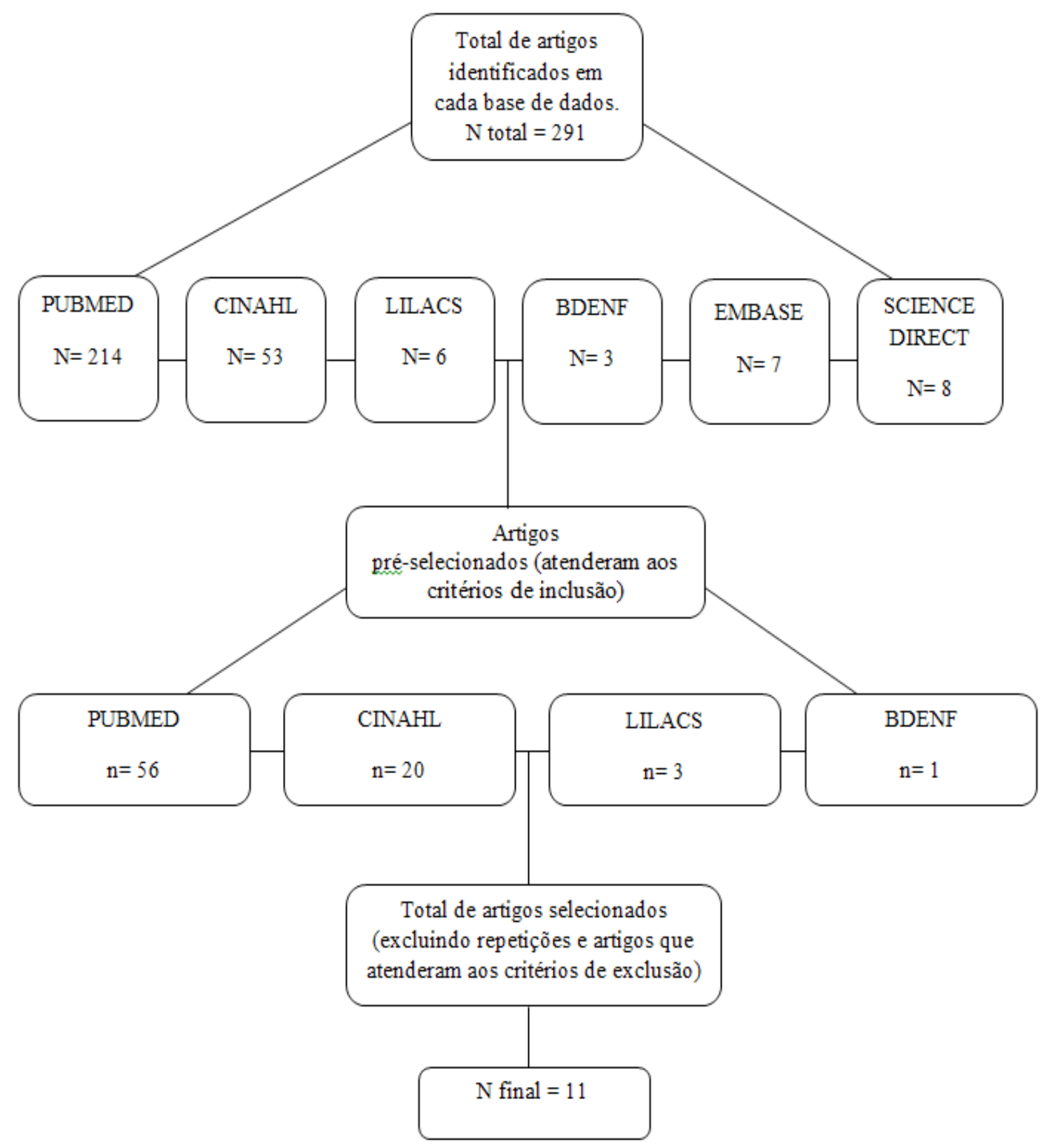

Figura 1 - Quantificação de artigos encontrados nas bases de dados pesquisadas e fluxograma para chegar-se à amostra do estudo. Redenção, CE, Brasil, 2016.

Procedeu-se a leitura e análise dos artigos, categorizando-os a partir das seguintes características: autores, objetivos, população e tamanho da amostra, conforme a Figura 2.

\begin{tabular}{|l|c|c|}
\hline \multicolumn{1}{|c|}{ Autores } & Objetivos & População e amostra \\
\hline Mesquita et al. & $\begin{array}{c}\text { Investigar o uso do enfrentamento religioso/espiritual por pessoas } \\
\text { com câncer, em quimioterapia. }\end{array}$ & $\begin{array}{c}101 \text { pacientes com câncer em } \\
\text { quimioterapia. }\end{array}$ \\
\hline $\begin{array}{l}\text { Pinto; Caldeira; } \\
\text { Martins. }\end{array}$ & $\begin{array}{c}\text { Analisar os níveis de espiritualidade dos pacientes oncológicos em } \\
\text { quimioterapia. }\end{array}$ & $\begin{array}{c}92 \text { pacientes com câncer em } \\
\text { quimioterapia. }\end{array}$ \\
\hline Guerrero et al. & $\begin{array}{c}\text { Compreender a relação entre espiritualidade e o enfrentamento ao } \\
\text { câncer na perspectiva dos pacientes oncológicos. }\end{array}$ & $\begin{array}{c}14 \text { pacientes com câncer em } \\
\text { quimioterapia. }\end{array}$ \\
\hline Balboni et al. & $\begin{array}{c}\text { Examinar a religiosidade e apoio espiritual em pacientes com câncer } \\
\text { avançado e a sua associação com a qualidade de vida. }\end{array}$ & $\begin{array}{c}230 \text { pacientes com falência da } \\
\text { quimioterapia. }\end{array}$ \\
\hline $\begin{array}{l}\text { Gaston-Johansson } \\
\text { et al. }\end{array}$ & $\begin{array}{c}\text { Examinar o enfrentamento, aflição psicológica, bem estar espiritual e } \\
\text { estratégias de enfrentamento de mulheres afroamericanas. }\end{array}$ & $\begin{array}{c}17 \text { mulheres afroamericanas com } \\
\text { câncer de mama. }\end{array}$ \\
\hline Canada et al. & $\begin{array}{c}\text { Investigar o papel da religião/espiritualidade e do enfrentamento na } \\
\text { qualidade de vida de mulheres em quimioterapia. }\end{array}$ & $\begin{array}{c}129 \text { mulheres com câncer de ovário } \\
\text { em quimioterapia. }\end{array}$ \\
\hline
\end{tabular}


Figura 2 - continuação

\begin{tabular}{|l|c|c|}
\hline Batista; Mendonça. & $\begin{array}{c}\text { Estudar o nível de espiritualidade em pacientes oncológicos e } \\
\text { correlaciona-los com qualidade de vida. }\end{array}$ & $\begin{array}{c}33 \text { pacientes com confirmação de } \\
\text { câncer, em tratamento. }\end{array}$ \\
\hline Fouladi et al. & $\begin{array}{c}\text { Compreender os fatores que afetam a continuidade dos cuidados, do } \\
\text { diagnóstico ao tratamento, em pacientes com câncer de mama. }\end{array}$ & $\begin{array}{c}22 \text { mulheres submetidas a cirurgia e } \\
\text { quimioterapia. }\end{array}$ \\
\hline Lissoni et al. & $\begin{array}{r}\text { Investigar as relações existentes entre o perfil psicoespiritual, } \\
\text { ritmo circadiano de cortisol e número de linfócitos antes e após } \\
\text { quimioterapia e a eficácia da própria quimioterapia. }\end{array}$ & $\begin{array}{c}30 \text { pacientes admitidos para receber } \\
\text { quimioterapia. }\end{array}$ \\
\hline $\begin{array}{l}\text { Günüsen; Inan; } \\
\text { Üstün. }\end{array}$ & $\begin{array}{c}\text { Identificar as experiências de mulheres com câncer e os fatores } \\
\text { facilitadores do enfrentamento enquanto recebem quimioterapia. }\end{array}$ & $\begin{array}{c}11 \text { mulheres com câncer de mama } \\
\text { em quimioterapia. }\end{array}$ \\
\hline $\begin{array}{l}\text { Caldeira; Carvalho; } \\
\text { Vieira. }\end{array}$ & $\begin{array}{c}\text { Avaliar o bem-estar espiritual de idosos com câncer submetidos à } \\
\text { quimioterapia, e o diagnóstico angústia espiritual. }\end{array}$ & $\begin{array}{c}45 \text { pacientes com câncer submetidos } \\
\text { a quimioterapia. }\end{array}$ \\
\hline
\end{tabular}

Figura 2 - Síntese dos artigos publicados nas bases de dados no período de 2005 a 2016. Redenção, CE, Brasil, 2016.

\section{Resultados}

Onze artigos atenderam aos critérios de inclusão estabelecidos. As publicações predominaram na base de dados PUBMED (6), seguida por LILACS (3) e CINHAL e BDENF, ambos (1). As bases ScienceDirect e EMBASE não tiveram artigos selecionados no $\mathrm{N}$ final.

Quanto ao desenho metodológico, os estudos tiveram abordagens variadas, a saber: misto (4), quantitativo (4) e qualitativo (3). O idioma de publicação predominante foi o inglês (6), seguido pelo português (5).

Os estudos foram desenvolvidos no Brasil (4), Estados Unidos (3), seguidos pelos países Irã, Itália, Portugal e Turquia com 1 estudo cada.

A maioria dos estudos foi publicada nos últimos cinco anos, a saber: 2013 (3), 2014 (2), 2012 (2), ademais, em 2011, 2008, 2007 e 2006 todos com apenas uma publicação cada.

Evidenciou-se que os artigos encontrados na busca, em sua maioria (5), foram desenvolvidos por equipe multiprofissional, seguidos por (4) estudos realizados por enfermeiros e (2) por médicos.

$\mathrm{Na}$ avaliação do enfrentamento religiosos/ espiritual em sessões de quimioterapia, verificouse que boa parte (7) dos artigos utilizaram instrumentos validados como a Escala da Avaliação da Espiritualidade em Contextos de Saúde (EAECS), Escala de Coping Religioso-Espiritual (CRE), Brief RCOPE, Questionário de Bem Estar Espiritual (QBEE), Functional Assesment of Cancer Therapy General (Fact-G) e Functional Assesment of Chronic Illness Therapy-Spiritual Well-Being Scale (FacitSp 12). Observou-se, ainda, que os instrumentos repetiram-se em alguns estudos.

O auxílio da religiosidade/espiritualidade para o enfrentamento do câncer, durante tratamento quimioterápico, foi um consenso entre os artigos científicos incluídos nesta revisão integrativa.
Observa-se que essa temática é de interesse de vários profissionais da área da saúde, ressaltando assim a importância de pesquisas sobre pessoas com câncer e o enfrentamento religioso/espiritual.

\section{Discussão}

Na última década, uma atenção maior tem sido dispensada ao estudo da espiritualidade/religiosidade como estratégia de enfrentamento, utilizada por pessoas com câncer, frente ao seu papel protetor contra a morbidade psicológica. Cada indivíduo relaciona a espiritualidade à esperança de sobreviver ao câncer ${ }^{(5)}$.

Após a leitura dos artigos, extração e análise das informações pertinentes para responder a pergunta norteadora, as informações foram organizadas em três categorias: utilização do CRE positivo ou negativo pelos pacientes em quimioterapia; relação do CRE e a prática religiosa e CRE associado com a qualidade de vida e bem-estar espiritual do paciente em quimioterapia.

\section{Utilização do CRE positivo ou negativo pelos pacientes em quimioterapia}

A vivência do sofrimento causado pela doença desencadeia uma conexão maior com a religiosidade e a espiritualidade, o que favorece escore alto de CRE positivo ${ }^{(2,11-14)}$. O papel da religiosidade, neste caso, pode estar relacionado com questões como resiliência, esperança e bem estar espiritual. Além disso, a religiosidade coopera no manejo da ideia do câncer como uma doença de caráter divino(4,11,14).

Existem evidências de que pessoas com algum tipo de espiritualidade tendem a apresentar menor incidência de doenças, maior longevidade, recuperação mais rápida e menos intercorrências durante o tratamento. Porém, a religião/espiritualidade pode ser fonte de desconforto e estresse, a depender 
de como a pessoa se relaciona com ela, ou seja, se utiliza estratégias de CRE positivo ou negativo(15).

Fato é que o CRE negativo está correlacionado ao aumento da incidência de efeitos colaterais, maior presença de sofrimento psicológico com consequente piora da saúde mental, ansiedade, menor uso de autoafirmações positivas, depressão, além de piores níveis de qualidade de vida e menos bem-estar espiritual total. O CRE negativo é mais característico em indivíduos mais jovens, sem religião e que desconsideram o apoio espiritual|(5,11,15-16).

\section{Relação do CRE e a prática religiosa}

Pessoas com alguma religião organizada e cuja prática religiosa é regular apresentam médias de espiritualidade superior, resultando no uso do CRE de forma mais positiva ${ }^{(2,5,11,13-14)}$.

Dentre as razões para uma associação positiva entre religião e saúde está o fato de que crenças e práticas religiosas podem evocar emoções positivas. Assim, considerar a religião/espiritualidade como algo importante na vida evidencia também que os indivíduos que professam alguma fé, e tem religião declarada, apresentam média de CRE negativo irrisório(12).

Uma particularidade destacável é o apoio da Religiosidade/espiritualidade no campo da psiconeuroendocrinologia. Alguns autores já averiguaram boa resposta à quimioterapia, nos níveis de linfócitos e cortisol e no tamanho do tumor, em pessoas com bons escores em testes de espiritualidade $^{(17)}$. Este mecanismo de apoio pode ser maior em idosos, pois, esses são mais crentes e/ou possuem uma visão menos materialista e mais transcendental em relação à vida. Isto é importante ainda por que os idosos com câncer sofrem mais com angústia espiritual(16).

CRE relacionado com a qualidade de vida e bem estar espiritual entre pessoas em quimioterapia

O CRE e o apoio das necessidades espirituais contribuem de forma positiva para melhorar a qualidade de vida dos pacientes em quimioterapia, através do aumento do bem-estar pisicológico, emocional, funcional e afetivo dos pacientes ${ }^{(5,12,15-16,18)}$.

Talvez em virtude disso, autores tenham averiguado o desejo de pacientes de receber algum tipo de cuidado espiritual durante a quimioterapia. Entretanto, a maioria queixa-se de não terem suas necessidades espirituais contempladas durante 0 tratamento ${ }^{(5,18)}$.
Inúmeros obstáculos impedem que enfermeiros contribuam com a prestação de apoio espiritual, simultaneamente ao cuidado biológico. A falta de formação profissional e de certa forma a carência teórica para o cuidado espiritual apresentam-se como pretexto para a deficiência da fornecimento do mesmo.

No entanto, a Nursing Interventions Classification (NIC), que se apresenta como um referencial consolidado acerca das intervenções de enfermagem, contém duas intervenções relacionadas especificamente à espiritualidade: apoio espiritual e facilitação do crescimento espiritual. Ao implementar essas intervenções, a enfermagem atende as necessidades espirituais do paciente, realizando a interface entre a espiritualidade e o corpo de conhecimento próprio da profissão(2).

Fato é que, na visão de alguns pacientes oncológicos, o enfermeiro é o profissional com maior habilidade para lidar com pessoas em processo de sofrimento espiritual, por terem maior disponibilidade para escuta e demonstrarem mais carinho no cuidado ${ }^{(14)}$.

\section{Considerações finais}

Os resultados obtidos demonstram que 0 paciente oncológico deve ser visto em sua totalidade, e que seus aspectos religiosos/espirituais devem ser compreendidos, valorizados e considerados para um cuidado de saúde individualizado e singular. Esses achados possibilitam-nos refletir sobre a importância da espiritualidade na vida das pessoas em tratamento quimioterápico, sendo essa fundamental para o enfrentamento da doença.

Cabe destacar que o CRE pode apresentarse como elemento que contribui na adesão ao tratamento e na redução do estresse situacional e da ansiedade. Respeitar a crença do indivíduo, e considerá-la, contribui também para uma melhor relação interpessoal entre a equipe profissional e a pessoa cuidada.

Portanto, o estudo traz contribuições relevantes para a enfermagem, tendo em vista que o enfermeiro é o profissional que se destaca diante de sua maior proximidade na rotina do paciente em tratamento quimioterápico, tornando-se referência para o estabelecimento do vínculo de confiança, facilitando assim, o fornecimento do apoio espiritual para essas pessoas que podem estar fragilizadas espiritualmente.

Uma das limitações apresentadas nesta revisão refere-se a quantidade de descritores controlados, que apresentaram-se em menor número do que os 
usualmente utilizados em revisões integrativas. Foram selecionados apenas dois descritores padronizados em todas as bases de dados. Acrescentar mais descritores em algumas bases de dados específicas poderia ter aumentado o número de estudos encontrados.

A partir deste estudo, criam-se, também, expectativas e possibilidades para o desenvolvimento de novas pesquisas que apontem para a necessidade de capacitação de profissionais da área da saúde para lidar com temas como a religiosidade/espiritualidade relacionados ao cuidado prestado aos pacientes oncológicos.

\section{Referências}

1.Instituto Nacional de Câncer José Alencar Gomes da Silva. Estimativa 2016: incidência de câncer no Brasil. Rio de Janeiro: INCA; 2015.

2. Guerrero GP, Zago MMF, Sawada NO, Pinto MH. Relação entre espiritualidade e câncer: perspectiva do paciente. Rev Bras Enferm. 2011;64(1):53-9.

3. Brateibach V, Domenico EBL, Berlezi EM, Loro MM, Rosanelli CLSP, Gomes JS, et al. Sintomas de pacientes em tratamento oncológico. Rev Ci Saúde. 2013 maiago;6(2):102-9.

4. Pinto S, Caldeira S, Martins JC. A espiritualidade em pacientes com câncer em quimioterapia. CuidArte Enferm. 2012;6(1):8-14.

5. Mesquita AC, Chaves ECL, Avelino CCV, Nogueira DA, Panzini RG, Carvalho EC. The use of religious/ spiritual coping among patients with cancer undergoing chemotherapy treatment. Rev. Latino-Am. Enfermagem. 2013;21(2). doi: http://dx.doi.org/10.1590/S010411692013000200010.

6. Felipe AOB, Carvalho AMP, Andrade CUB. Spirituality and religion as protectors for adolescent drug use. SMAD, Rev. Eletrôn Saúde Mental Álcool Drog. 2015;11(1):4958. doi: http://dx.doi.org/10.11606/issn.1806-6976. v11i1p49-58.

7. Brown AJ, Sun CC, Urbauer D, Zhukovsky DS, Levenback $\mathrm{C}$, Frumovitz $\mathrm{M}$, et al. Targeting those with decreased meaning and peace: a supportive care opportunity. http://link.springer.com/journal/520. 2015;23(7):2025-32.

8.Fornazari SA, Ferreira RER. Religiosidade/ Espiritualidade em Pacientes Oncológicos: Qualidade de Vida e Saúde. Psicologia: Teoria e Pesquisa. 2010;26(2):265-72.

9. Barlow F, Walker J, Lewith G. Effects of Spiritual Healing for Women Undergoing Long-Term Hormone Therapy for Breast Cancer: A Qualitative Investigation. J
Alternative Compl Med. 2013;19(3):211-6.

10. Paula W Jr, Zanini DS. Pacientes em radioterapia: um estudo de coping. Psicol Saúde Doenças. 2012;13(2):480-93.

11. Gaston-Johansson F, Haisfield-Wolfe ME, Reddick B, Goldstein N, Lawal TA. The Relationships Among Coping Strategies, Religious Coping, and Spirituality in African American WomenWith Breast Cancer Receiving Chemotherapy. Oncol Nurs Forum. 2013;40(2):120-31.

12. Canada AL, Parker PA, de Moor JS, Basen-Engquist $\mathrm{K}$, Ramondetta LM, Cohen L. Active coping mediates the association between religion/spirituality and quality of life in ovarian cancer. Gynecol Oncol. 2006;101:102-7.

13. Fouladi N, Ali-Mohammadi H, Pourfarzi1 F,Homaunfar N.Exploratory Study of Factors Affecting Continuity of Cancer Care: Iranian Women's Perceptions. Asian Pac J Cancer Prev. 2014;15(1):133-7.

14. Günüşen NP, İnan FS, Üstün B. Experiences of Turkish Women with Breast Cancer During the Treatment Process and Facilitating Coping Factors. Asian Pacific J Cancer Prev. 2013;14(5):3143-9.

15. Batista S, Mendonça ARA. Espiritualidade e qualidade de vida nos pacientes oncológicos em tratamento quimioterápico. Rev Bioética. 2012;20(1):175-88.

16. Caldeira S, de Carvalho EC, Vieira M. Between spiritual wellbeing and spiritual distress: possible related factors in elderly patients with cancer. Rev. Latino-Am. Enfermagem. 2014;22(1):28-34.

17. Lissoni P, Messina G, Balestra A, Colciago M, Brivio F, Fumagalli $L$, et al. Efficacy of Cancer Chemotherapy in Relation to Synchronization of Cortisol Rhythm, Immune Status and Psychospiritual Profile in Metastatic Nonsmall Cell Lung Cancer. In Vivo. 2008;22:257-2.

18. Balboni TA, Vanderwerker LC, Block SD, Paulk ME, Lathan CS,Peteet JR, et al. Religiousness and Spiritual Support Among Advanced Cancer Patients and Associations With End-of-Life Treatment Preferences and Quality of Life. J Clin Oncol. 2007;25(5):555-60. 